\title{
Molecular Cloning of Leishmania majorgp63 Gene in BALB/c Mouse CT26 Cell Line
}

\author{
Hossein Rezvan ${ }^{1, *}$ \\ ${ }^{1}$ Department of Laboratory Sciences, Faculty of Paraveterinary Sciences, Bu-Ali Sina University, Hamadan, IR Iran \\ ${ }^{*}$ Corresponding author: Hossein Rezvan, Department of Laboratory Sciences, Faculty of Paraveterinary Sciences, Bu-Ali Sina University, Hamadan, IR Iran. E-mail: hrezvan@gmail.com
}

Received: January 16, 2014; Accepted: April 5, 2014

\begin{abstract}
Background: Leishmaniasis is now accounted as a worldwide disease, which is caused by different species of Leishmania parasite. This parasitic disease is now endemic in many areas including 16 developed and 72 developing countries. An estimated 12 million cases of leishmaniasis exist worldwide and a further 367 million are at the risk of acquiring the disease. A high rate of Leishmania and HIV coinfection has now been reported from 30 countries around the world. Th1 immune responses have the main role in eliciting immunity to Leishmania parasite. Many attempts have been made and different strategies have been approached to develop a potent vaccine against this parasite.

Objectives: The main objective of this study was to clone and detect the gene encoding Leishmania major gp63 in mouse CT26 cell line. Materials and Methods: In this observational study, the Leishmania major gp63 gene segment was amplified using specific primers and then sub-cloned into pcDNA3 expression vector. The new construct was transfected into mouse CT26 cell line.

Results: The results clearly showed that pcDNA3 L. major gp63 was successfully constructed and transfected into CT26 mouse cell line and these cells have a high potency in accepting and expressing Leishmania genes.

Conclusions: A full length of L. major gp63 gene was cloned in to mouse CT26 cell line, which can be used in future vaccine studies for Leishmania.
\end{abstract}

Keywords: Leishmania major; gp63; Cloning; BALB/c

\section{Background}

Leishmaniasis as a parasitic disease is now considered as a neglected disease by WHO in many countries around the world. However, the high incidence rate of the disease occurs in tropical and temperate regions where the Leishmania vector lives [1]. According to WHO, the number of leishmaniasis cases has been increasing during last two decades where more sensitivity with a high rate of Leishmania and AIDS co-infection cases is reported in patients suffering from AIDS in many areas [2-5]. Chemotherapy against Leishmania infestation, because of the toxicity and drug resistance, is not totally worthwhile [6, 7]. It has been shown that Th1 immune responses are crucial in immunity to Leishmania parasite [8] and because of the capability of single antigenic proteins in inducing Th1 immune responses, these proteins are now in main focus of vaccine candidates against the pathogen $[9,10]$.

Vaccination is postulated as the most appropriate opportunity for the prevention and safe treatment of all forms of the disease so developing a safe, effective and affordable anti-leishmanial vaccine is accounted as a promising approach for Leishmania researchers [11].

Several studies have been carried out to prove the immunogenicity of killed Leishmania parasite using different adjuvants such as Bacillus Calmette Guerin (BCG) [12] but no significant protection was observed following the in- jection of the vaccine. However, the mixture was safe and induced Leishmania Skin Test (LST) conversion with weak but measurable IFN- production. Using live Leishmania as a potent vaccine (leishmanization) has been considered as another strategy in the prevention of leishmaniasis [13]. Although, the live vaccine is of low cost and highly immunogenic, it still lacks standardization and quality control.

Genetically modified Leishmania is carried out either by mutagenesis and or gene targeting methods in which either a foreign gene is introduced into the parasite genome or by knocking out virulency genes [14]. Recently, recombinant proteins of Leishmania species have been used to produce the immunity against different species of Leishmania. Different proteins have been tested to be used as a vaccine including recombinant acylated surface protein B1 (HASPB1) and promastigote surface antigen-2 (PSA-2), which are immunogenic and can develop variable levels of immunity in different animal models.

Leishmania gp63 or leishmanolysin, an immunogenic Leishmania protein, is shown to be present in all Leishmania species and accounted as a good vaccine candidate in Leishmania studies $[15,16]$. Injection of gp63 in saline, Complete Freud's Adjutant(CFA), Bacillus Calmette Guerin (BCG) and Corynebacterium parvum induced significant protection in mice. So, cloning Leishmania gp63 into a

Copyright (c) 2015, Zahedan University of Medical Sciences. This is an open-access article distributed under the terms of the Creative Commons Attribution-NonCommercial 4.0 International License (http://creativecommons.org/licenses/by-nc/4.0/) which permits copy and redistribute the material just in noncommercial usages, provided the original work is properly cited. 
BALB/ mouse cell line would be a feasible strategy in developing a potent cell line expressing an immunogenic Leishmania protein to be used for Leishmania vaccines.

\section{Objectives}

The main objective of this study was to clone and detect the gene encoding L. major gp63 in mouse CT26 cell line.

\section{Materials and Methods}

In this observational study, $\mathrm{N}$-methylurethane-induced BALB/c murine colon carcinoma (CT-26) cells were gifted by Prof Ian Hart (St Thomas hospital) and cultured in Dulbecco's Modified Eagle's Medium (DMEM) + 10\% Fetal Calf Serum (FCS).

\subsection{Preparation of L. major cDNA}

CMV promoter pNUT L. major gp63 DNA was kindly gifted by Prof Mack Master (Department of Medical Genetics, University of British Columbia, Vancouver, Canada). The construct was first sequenced by MWG-Biotech using the primers ATCACGAAGTCGGTGTTG, GCACATCACCGAGGGCTT and AGAGCGAGGACGCCATCC and checked for mismatches against the sequence of the gene (code number Y00647) in the gene bank.

\subsection{PCR Amplification}

PCR was performed using a DNA thermal cycler (Thermo Hybaid, USA) for amplification of the whole gene. Primers were supplied by Sigma Genosys (UK). For amplification by PCR, $1 \mu \mathrm{L}$ of cDNA was mixed with $5 \mu \mathrm{L}$ of $10 \times$ PCR buffer, $0.8 \mu \mathrm{L}$ each of $10 \mathrm{mmol} \mathrm{dNTP}, 20 \mathrm{pmol}$ each of primer solutions, 1.25 unit of thermostable Taq polymerase (Bioline), $1.5 \mathrm{mmol} \mathrm{MgCl} 2$ (Bioline) and then water was added to a total volume of $50 \mu \mathrm{L}$. The PCR program was set up as an initial melting step at $95^{\circ} \mathrm{C}$ for 10 minutes followed by 35 cycles including 1 minute at $95^{\circ} \mathrm{C}$ for denaturation, 45 seconds at $58^{\circ} \mathrm{C}$ for annealing and 2 minutes or $45 \mathrm{sec}-$ onds (only for screening) at $72^{\circ} \mathrm{C}$ for extension and a final extension step at $72^{\circ} \mathrm{C}$ for 10 minutes. PCR products were visualized using a $1.5 \%(\mathrm{w} / \mathrm{v})$ agarose gel containing $1 \mu \mathrm{g} /$ $\mathrm{mL}$ of ethidium bromide (BDH Laboratories, UK).

\subsection{Ligation of L. major gp63 Gene and pcDNA3 Vector}

The L. major gp63 gene produced by PCR using the primers containing HindIII and EcoRI restriction sites and the pcDNA3 vector were digested with relevant restriction enzymes and run onto the gel agarose. The gene and the vector were then cut and extracted from the gel by DNA extraction kit (Gene Flow) with accordance to the manufacturer's protocol and ligated together using ligation enzyme (promega). The ligation was set up by adding 0.5 $\mu \mathrm{L}$ ligation enzyme, $1 \mu \mathrm{L}$ buffer and $6.5 \mu \mathrm{L}$ water to $2 \mu \mathrm{L}$ of each DNA (adjusted at $10 \mu \mathrm{L}$ ). The ligated DNA was incubated at $4^{\circ} \mathrm{C}$ overnight. The pcDNA3 vector containing $L$. major gp63 gene was reproduced by transforming E. coli and extracting the DNA from the bacteria.

\subsection{Antibiotic Sensitivity Assay}

In presence of a serial concentration of Geneticin (G418) (Sigma) from 50 to $900 \mu \mathrm{g} / \mathrm{mL}, 1 \times 10^{6} /$ well CT26 tumour cells were cultured in 24-well plates; wells for each concentration of the antibiotic were put in duplicate. The cells were incubated at $37^{\circ} \mathrm{C}$ with $0.5 \mathrm{CO}_{2}$ for 10 days. The lower concentration $(500 \mu \mathrm{g} / \mathrm{mL})$ of the antibiotic in which all the CT26 tumour cell died in 7-10 days was chosen for transfection.

\subsection{Transfection of CT26 Tumour Cells With L. ma- jor gp63}

Lipofectamine 2000 (Invitrogen) was used to transfect CT26 tumour cells with pcDNA3 L. major gp63. Briefly, the culture of CT26 tumour cells at $1 \times 10^{6}$ per well in 24-well plates with $90 \%$ confluence was chosen. pcDNA3 L. major gp63 and lipofectamine 2000 were diluted in serum free DMEM media at $0.8 \mu \mathrm{g} / 50 \mu \mathrm{L}$ and $2 \mu \mathrm{g} / 50 \mu \mathrm{L}$ respectively and incubated for 5 minutes at room temperature. The diluted DNA and lipofectamine 2000 were mixed together and incubated again at room temperature for 20 - 30 minutes. After removing the supernatant, the DNA-lipofectamine was added to the CT26 cell culture and the culture was incubated at $37^{\circ} \mathrm{C}$ with $0.5 \% \mathrm{CO}_{2}$ for $4-6$ hours. One milliliter per well DMEM media containing 10\% FCS was then added to the culture. Sixteen until 24 hours later, the media was replaced with a fresh media complemented with $10 \%$ FCS and $500 \mu \mathrm{g} / \mathrm{mL}$ G418.

\subsection{Screening the Expression of L. major gp63 Gene by RT-PCR}

The stable transfected CT26 tumour cells were screened for the presence of the L. major gp63 gene by RT-PCR. Total RNA was isolated from the cells using RNA STAT-60 (AMS Biotechnology, UK) following manufacturer's instructions. Briefly, CT26 L. major gp63 were cultured in T25 tissue culture flasks. After removing the media, $1 \mathrm{~mL}$ of RNA-STAT60 was added to the cell culture followed by incubating at room temperature for 5 minutes, adding 0.2 $\mathrm{mL}$ of chloroform, vigorously shaking for 60 seconds and leaving at room temperature for 3 minutes. The homogenate was then centrifuged at 14,000 rpm for 10 minutes and the aqueous phase was separated. Total of $0.5 \mathrm{~mL}$ of isopropanol was added the aqueous phase of samples followed by 8 minute incubation at room temperature and 15 minute centrifugation at 14,000 rpm. After removing the supernatant, the RNA pellet was washed with $75 \%$ ethanol, dried and resuspended in molecular grade water.

\subsection{RNA was Then Reverse Transcribed into cDNA as Follow}

Two microgram of RNA and $0.5 \mu$ g of oligo (dT15) primer 
(Biorad) were heated at $70^{\circ} \mathrm{C}$ for 5 minutes and placed on ice. The following mixture was then added to the tube: Five microliter of $5 \times$ reaction buffer, $1 \mu \mathrm{L}$ of dNTPs $(12.5 \mathrm{mmol})$ (Biorad), 25 units rRNasin Ribonuclease Inhibitor (Biorad), 200 units of M-MLV Reverse Transcriptase (Biorad), nuclease free water was added to make the final volume to 25 $\mu \mathrm{L}$. Contents of the tube were gently mixed and heated at $39.2^{\circ} \mathrm{C}$ for 80 minute followed by cooling on ice and heating at $95^{\circ} \mathrm{C}$ for 5 minute and then storing them at $20^{\circ} \mathrm{C}$. PCR amplification was applied to screen the presence of $L$. major gp63 gene using the reverse transcribed RNA as template.

\section{Results}

\subsection{L. major gp63 DNA Sequencing}

The sequence of the gene was evaluated (Figure 1) using specific primers and aligned with the sequence of the gene in the gene bank (see materials and methods). CMV promoter pNUT L. majorgp63 DNA(Figure 2) was first bulked up by transformation of E. coli.

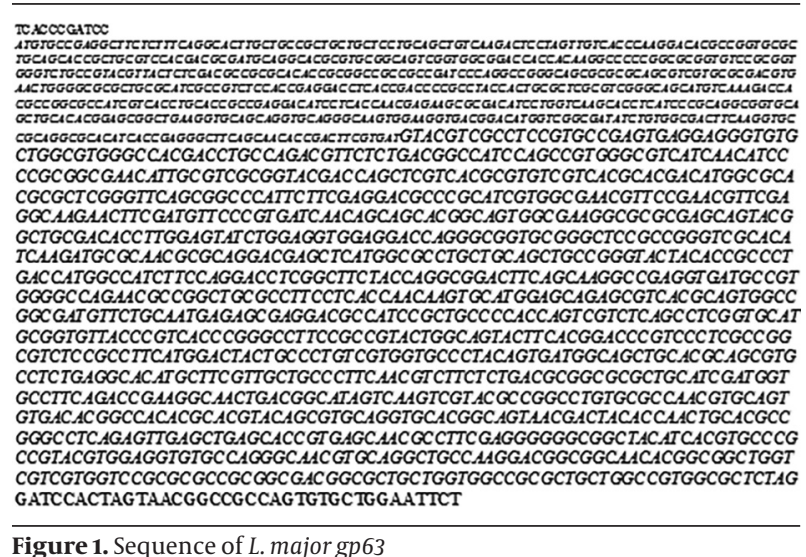

Figure 1. Sequence of L. major gp63

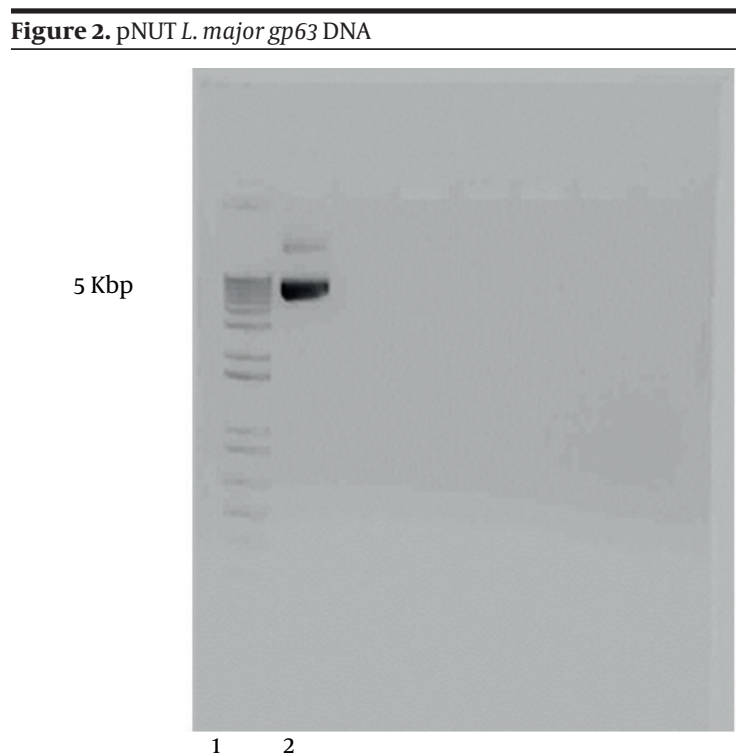

1) standard DNA, 2) L. major gp63.

\subsection{L. major gp63 Gene Sub-Cloning}

L. major gp63 gene was copied by PCR using two specific primers (5'- TCTAAGCTTCACCCGATCCATG TGCCG-3' and 5'- AGAATTCCTGCACACTGGCGG CCGTTA -3') in which two restriction sites HindIII and EcoRI were engendered. The copied gene and pcDNA3 vector (Figure 3) were both cut by the relevant enzymes and the digested products were run onto the gel agarose (Figure 4). L. major gp63 and pcDNA3 bands were cut off the gel and the DNAs were extracted. The enzyme digested pcDNA3 vector and the $L$. major gp63 DNA were ligated together (see materials and methods). After ligation the existence of the gene in pcDNA3 vector were checked and the gene was completely sequenced and aligned with that of pNUT vector. No mismatches were found between the two sequences.

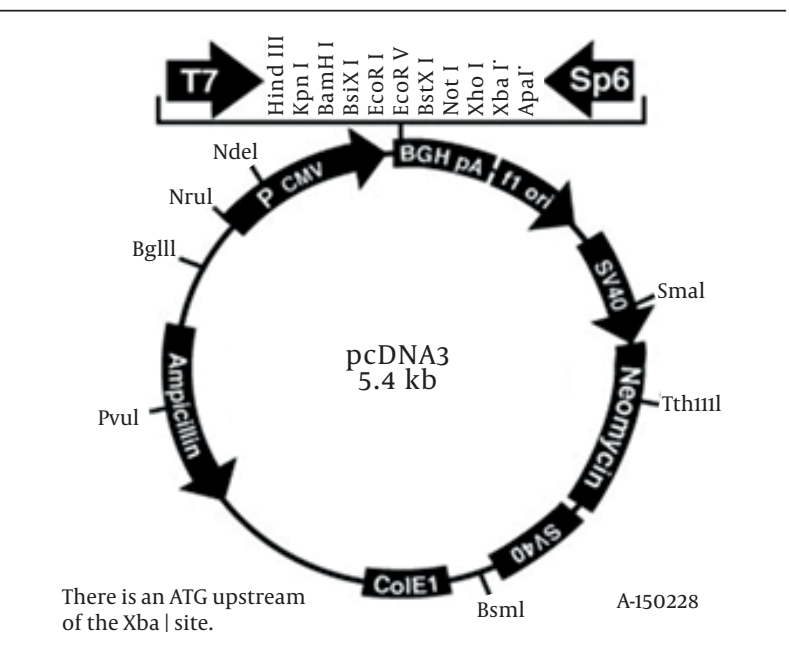

Figure 3. pcDNA3 Vector Map

Figure 4. pcDNA3 and L. major gp63 gene cut by HindIII and EcoRI restriction enzymes

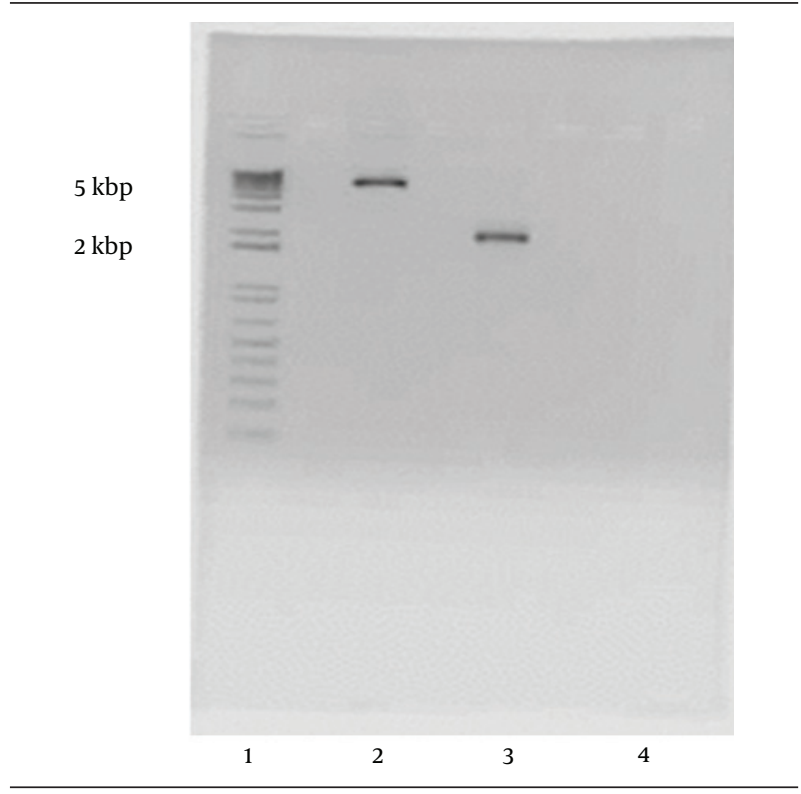

1: standard DNA, 2: PCR control, 3: pcDNA3, 4: L. major gp63. 


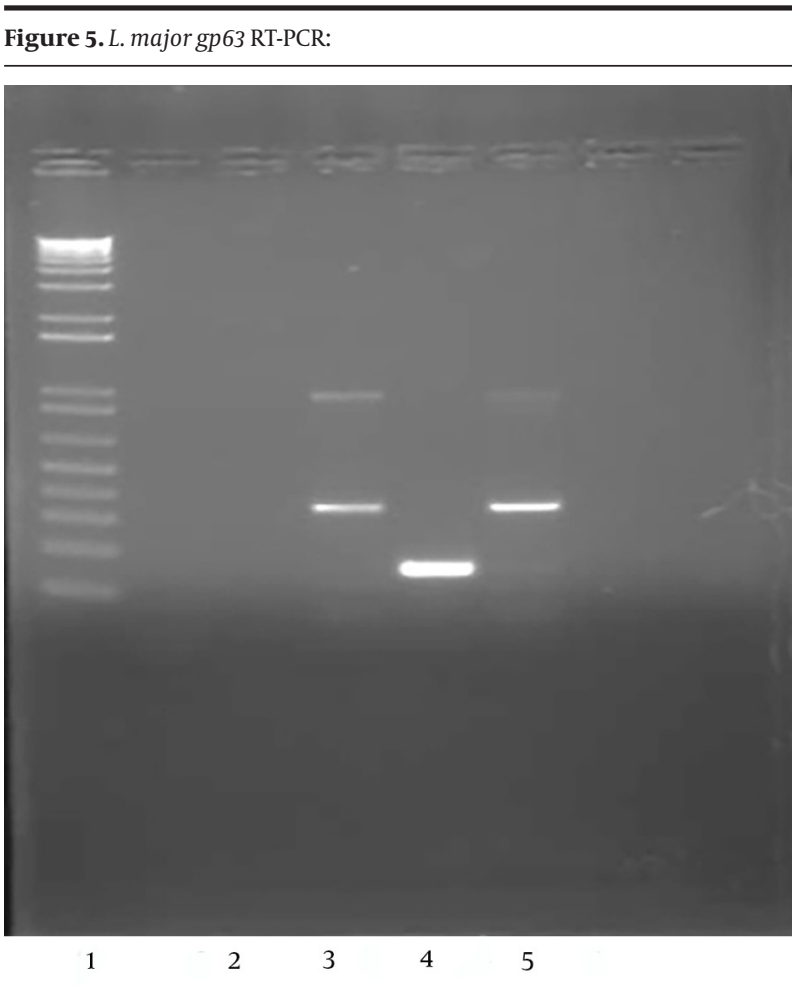

1: standard DNA, 2: PCR control, 3, 5: mouse GAPDH, 4: L. major gp63 cDNA.

\subsection{Transfection of CT26 Tumour Cells With L. ma- jor gp63}

pcDNA3 L. major gp63 was applied onto CT26 tumour cells for transfection of the cells using lipofectamine 2000 (see materials and methods). The presence of the gene was examined with detection of its mRNA using RTPCR. The following mouse GAPDH primers 5'-ACTCCACTCACGGCAAATTC-3' and 5'-CCTTCCA CAATGCCAAAGTT-3' were used to check the efficacy of the mRNA extracted from the CT26 transfected cells and specific L. major gp63 primers used to check the presence of the gene (Figure 5).

\section{Discussion}

The results of our study clearly showed that Leishmania gp63 successfully transfected into CT26 mouse cell line and these cells have a high potency in accepting Leishmania genes and expressing the parasitic proteins.

Developing specific resistance after infection approves the feasibility of vaccine approaches against Leishmania [17]. Different attempts have been made to generate Leishmania vaccines using recombinant immunogenic Leishmania proteins expressed in microbial vectors but none has yet been introduced as a vaccine for Leishmania $[18,19]$. However, the potency of mammalian cells in expression of Leishmania immunogenic genes is not yet fully cleared.

Cloning and characterization of antigenic Leishma- nia genes is now postulated as a promising approach in Leishmania vaccination. So far, different studies have been conducted to clone, express or manipulated Leishmania genes. Cloning and characterization of a gene encoding class I nuclease in L. infantum has already been carried out by Farajnia et al. [20]. Also, there are other reports on cloning and expression of different Leishmania genes such as HSP70 and glucose-regulated protein 94 (GRP94) gene from L. infantum [21, 22], surface protein encoding genes from L. major $[23,24]$ and L. donovani avastin gene [25]. Some other researchers tried to find similarity between genes cloned in other protozoa and the Leishmania ones in order to develop immunity against Leishmania [26]. There are some attempts for establishing cell lines specific for Leishmania, which were promising [17]. One of the characterised immunogenic proteins in all Leishmania species is Leishmania gp63 or leishmanoly$\sin [16]$. It has been shown that vaccination based on gp63 DNA or recombinant gp63 protein is potential to induce T cell stimulation against Leishmania in human producing high levels of INF- from effector T cells [27, 28]. Our previous studies also proved the immunogenicity of peptides predicted for either class I or II derived from this protein $[29,30]$. We also showed that expression of Leishmania gp63 protein in host's cells by either injection or using the gene gun can elicit immunity to Leishmania [31]. Similar results have been demonstrated for LACK antigen where in presence of recombinant IL-12 evokes CD4+ T cell inducing protection in mice against $L$. major infection [32]. Therefore, cloning Leishmania gp63 into a BALB/ mouse cell line would be a feasible strategy in developing a potent cell line expressing an immunogenic Leishmania protein to be used for Leishmania vaccines.

In present study, it was tried to clone a gene derived from protozoan cells and express it in mammalian cells in order to develop a potent mammalian cell line expressing an immunogenic Leishmania protein, which can be used for Leishmania vaccines. To clone the gene, L. major gp63 DNA was first cut from a week construct (pNUT vector) and sub-cloned into pcDNA3 vector using specific primers.

In conclusion, a full length of L. major gp63 gene was cloned into the mouse CT26 cell line and the stable expression of the gene was evaluated. The results confirmed that Leishmania genes can be successfully expressed in mammalian cell lines, which could be used as a potential vaccine against Leishmania.

\section{Acknowledgements}

We would like to thank the ministry of science, research and technology and BU-Ali Sina University for their financial support and the Nottingham Trent University for supplying the materials.

\section{Funding/Support}

This study was supported by Bu-Ali Sina University. 


\section{References}

1. Royer MA, Crowe CO. American cutaneous leishmaniasis. Arch Pathol Lab Med. 2002;126(4):471-3.

2. TDR/WHO . Leishmaniasis. 2012. Available from: http://www.who. int/tdr/diseases/leish/info/en/index.html.

3. Griekspoor A, Sondorp E, Vos T. Cost-effectiveness analysis of humanitarian relief interventions: visceral leishmaniasis treatment in the Sudan. Health Policy Plan. 1999;14(1):70-6.

4. Desjeux P. Leishmaniasis: current situation and new perspectives. Comp Immunol Microbiol Infect Dis. 2004;27(5):305-18.

5. Kar K. Serodiagnosis of leishmaniasis. Crit Rev Microbiol. 1995;21(2):123-52.

6. Soto J, Rojas E, Guzman M, Verduguez A, Nena W, Maldonado M et al. Intralesional antimony for single lesions of bolivian cutaneous leishmaniasis. Clin Infect Dis. 2013;56(9):1255-60.

7. Zucca M, Scutera S, Savoia D. New chemotherapeutic strategies against malaria, leishmaniasis and trypanosomiases. Curr Med Chem. 2013;20(4):502-26.

8. Ali SA, Rezvan H, McArdle SE, Khodadadi A, Asteal FA, Rees RC. CTL responses to Leishmania mexicana gp63-cDNA vaccine in a murine model. Parasite Immunol. 2009;31(7):373-83.

9. Maroof A, Brown N, Smith B, Hodgkinson MR, Maxwell A, Losch FO, et al. Therapeutic vaccination with recombinant adenovirus reduces splenic parasite burden in experimental visceral leishmaniasis. JInfect Dis. 2012;205(5):853-63.

10. Kedzierska K, Curtis JM, Valkenburg SA, Hatton LA, Kiu H, Doherty $\mathrm{PC}$, et al. Induction of protective CD4+ T cell-mediated immunity by a Leishmania peptide delivered in recombinant influenza viruses. PLoS One. 2012;7(3)

11. Mayrink W, Mendonca-Mendes A, de Paula JC, Siqueira LM, Marrocos Sde R, Dias ES, et al. Cluster randomised trial to evaluate the effectiveness of a vaccine against cutaneous leishmaniasis in the Caratinga microregion, south-east Brazil.Trans $R$ Soc Trop Med Hyg. 2013;107(4):212-9.

12. Armijos RX, Weigel MM, Aviles H, Maldonado R, Racines J. Field trial of a vaccine against New World cutaneous leishmaniasis in an at-risk child population: safety, immunogenicity, and efficacy during the first 12 months of follow-up.J Infect Dis. 1998;177(5):1352-7.

13. Khamesipour A, Rafati S, Davoudi N, Maboudi F, Modabber F. Leishmaniasis vaccine candidates for development: a global overview. Indian J Med Res. 2006;123(3):423-38.

14. Joshi PB, Sacks DL, Modi G, McMaster WR. Targeted gene deletion of Leishmania major genes encoding developmental stage-specific leishmanolysin (GP63). Mol Microbiol. 1998;27(3):519-30.

15. Chaudhuri G, Chaudhuri M, Pan A, Chang KP. Surface acid proteinase (gp63) of Leishmania mexicana. A metalloenzyme capable of protecting liposome-encapsulated proteins from phagolysosomal degradation by macrophages. J Biol Chem. 1989;264(13):7483-9.

16. Seay MB, Heard PL, Chaudhuri G. Surface Zn-proteinase as a molecule for defense of Leishmania mexicana amazonensis promastigotes against cytolysis inside macrophage phagolysosomes. Infect Immun. 1996;64(12):5129-37.

17. Arora SK, Pal NS, Mujtaba S. Leishmania donovani: identification of novel vaccine candidates using human reactive sera and cell lines. Exp Parasitol. 2005;109(3):163-70.

18. Soudi S, Hosseini AZ, Hashemi SM. Co-administration of rectal BCG and autoclaved Leishmania major induce protection in susceptible BALB/c mice. Parasite Immunol. 2011;33(10):561-71.

19. Ravindran R, Bhowmick S, Das A, Ali N. Comparison of BCG, MPL and cationic liposome adjuvant systems in leishmanial antigen vaccine formulations against murine visceral leishmaniasis. BMC Microbiol. 2010;10:181

20. Farajnia S, Rahbarnia L, Maleki Zanjani B, Alimohammadian $\mathrm{MH}$ Abdoli Oskoee S, Beh-Pajooh A, et al. molecular cloning and characterization of P4 nuclease from leishmania infantum. Enzyme Res. 2011;2011:970983.

21. Rasouli M, Zavaran Hoseini A, Kazemi B, Alborzi A, Kiany S. Expression of recombinant heat-shock protein 70 of MCAN/IR/96/ LON-49, a tool for diagnosis and future vaccine research. Iran J Immunol. 2009;6(2):75-86.

22. Larreta R, Soto M, Alonso C, Requena JM. Leishmania infantum: gene cloning of the GRP94 homologue, its expression as recombinant protein, and analysis of antigenicity. Exp Parasitol. 2000;96(2):108-15.

23. Cheng J, Zhong Y, Liu Y, Yang J. Cloning and sequence analysis of an amastin coding gene from Leishmania major Abdou. Chin Med J (Engl). 1999;112(8):698-700.

24. Chen J, Si CW, Wang QH, Liu Y, Zhong YW, Yang JZ, et al. [Cloning and sequence analysis of a gene encoding amastin from Leishmania major]. Chinese. Zhongguo Ji Sheng Chong Xue Yu Ji Sheng Chong Bing Za Zhi. 2000;18(1):30-2.

25. Li JF, Chen JP, Tian Y, Yang ZW, Ma Y, Hu XS. [Cloning of avastin gene of leishmania donovani isolates from Sichuan Province and its expression in eucaryotic system]. Chinese. Zhongguo $J i$ Sheng Chong Xue Yu Ji Sheng Chong Bing Za Zhi. 2007;25(2):124-8.

26. Bhardwaj S, Vasishta RK, Arora SK. Vaccination with a novel recombinant Leishmania antigen plus MPL provides partial protection against $\mathrm{L}$. donovani challenge in experimental model of visceral leishmaniasis. Exp Parasitol. 2009;121(1):29-37.

27. Mazumder S, Maji M, Ali N. Potentiating effects of MPL on DSPC bearing cationic liposomes promote recombinant GP63 vaccine efficacy: high immunogenicity and protection. PLoS Negl Trop Dis. 2011;5(12):e1429.

28. Mazumder S, Maji M, Das A, Ali N. Potency, efficacy and durability of DNA/DNA, DNA/protein and protein/protein based vaccination using gp63 against Leishmania donovani in BALB/c mice. PLoS One. 2011;6(2):e14644.

29. Rezvan H, Rees R, Ali S. Immunogenicity of MHC class I peptides derived from leishmania mexicana Gp63 in HLA-A2.1 transgenic (HHDII) and BALB/C mouse models. Iran J Parasitol. 2012;7(4):27-40.

30. Rezvan H. Immunogenicity of HLA-DR1 restricted peptides derived from Leishmania major gp63 using FVB/N-DR1 transgenic mouse model. Iran J Parasitol. 2013;8(2):273-9.

31. Rezvan H, Rees R, Ali S. Leishmania mexicana Gp63 cDNA using gne Gun induced higher immunity to L. mexicana infection compared to soluble Leishmania antigen in BALB/C. Iran J Parasitol. 2011;6(4):60-75.

32. Nagill R, Kaur S. Vaccine candidates for leishmaniasis: a review. Int Immunopharmacol. 2011;11(10):1464-88. 